\title{
Utilizing AR Glasses as Mobility Aid for People with Low Vision
}

\author{
Hein Min Htike* \\ School of Computer Science and Informatics, Cardiff University
}

\begin{abstract}
Augmented reality technology has the potential to improve what people with low vision can see by supplementing the real-world with visually accessible augmentations, thus providing useful information for mobility. This project aims to explore how AR can be used as a mobility aid for those people.
\end{abstract}

Keywords: Augmented reality, mobility aid, vision rehabilitation.

Index Terms: Human-centered computing-Human computer interaction (HCI)-Interaction paradigms-Mixed / augmented reality;

\section{INTRODUCTION}

A survey from National Center for Health Statistics (2007) [2] reported that nearly 27 million adults in the US are experiencing blindness or low vision, where one's vision cannot be corrected through glasses or by any medical or surgical treatments. Low vision can be categorized into central vision loss (CVL), peripheral field loss (PFL), or a combination of both, with approximately $74 \%, 13 \%$, and $11 \%$ of people with low vision (PLV) suffering from each type respectively. One of the significant problems they face, which has a severe impact on their quality of life, is reduced mobility. For these people, navigation and wayfinding are challenging as they have difficulty spotting obstacles, edges of stairs, and changes in surface level, or knowing the distance of objects when they have relatively low contrast with their surroundings. A wide range of solutions has been used to alleviate this problem: for example, using guide dogs or long canes; or making the environment more visually accessible by marking hazards (e.g., edges of stairs, platforms) with bright colors, or using tactile paving. These methods are not practical in many scenarios, and most of them can be cost-prohibitive.

In recent years, much research has been done to improve mobility through the use of technology. The majority of these electronic mobility aids use various sensors (e.g., camera, depth sensors, etc.) to capture the environment and translate the spatial and visual information into alternative modalities such as auditory and vibrotactile. While such vision substitution techniques would be essential for people who are completely blind, the majority of PLV has some useful residual vision and prefer to use it to observe the environment [9]. Therefore, it would be more intuitive for them to make the best use of their remaining vision through vision enhancements.

Currently, one approach to providing vision enhancement is to render visual scenes in a simplified manner. It represents objects in different colors or intensities depending on object types or distances [4], or in lower resolution [8] to accommodate the poor visual function of PLV, to allow them to focus on important objects such as obstacles and to understand their distances in a scene. While this reduces visual clutter, it also removes other information that might have been useful for PLV, such as shadows or texture details,

\footnotetext{
*htikeh@cardiff.ac.uk
}

etc. Another approach is to overlay a minified edge or color image of the wider visual scene onto the center of the field of view to extend the visual field (VF), an important indicator of mobility performance. This technique, dubbed "vision multiplexing" [6], can improve VF, but how these augmentations impact mobility performance is yet to be studied. Other approaches include realtime edge enhancement to improve contrast sensitivity (CS) [3], and digital zooming [5]. The effects of current vision enhancements using head-mounted AR devices are mixed, with reduced mobility efficiency but improved obstacle detection and object recognition.

The existing literature regarding visual mobility aids does not mention the involvement of PLV during the design and implementation stages to capture their mobility needs. However, what PLV can see differ based on the underlying visual conditions. Therefore, one of the significant challenges of designing a visual mobility aid for PLV is to understand the different needs and to provide tailored solutions flexible enough to help varying levels of visual functions and conditions. Another challenge is the robustness and efficiency of algorithms to run in real-time on mobile devices such as AR glasses. This project aims to explore how visual augmentations in the AR environment can be used as visual enhancements to provide information necessary for safe mobility in PLV.

\section{Plan}

To address the challenges mentioned in the previous section, this project aims to contribute to the understanding of the requirements of PLV in the AR environment for mobility, to develop computer vision algorithms required to generate appropriate vision augmentations, and to evaluate their effectiveness in mobility trials using HoloLens. HoloLens was chosen for its hardware capabilities, mature development tools, and ability to be worn on top of spectacles if necessary. The project will be conducted in three phases:

1. Formative study: to understand the mobility requirements of PLV (described further in the next section).

2. Software development: to identify potentially useful vision enhancements based on the analysis of literature and the outcome from the formative study and to develop software using participatory design approach [1] with continuous user involvement to ensure that the visual enhancements are fit for purpose. Developing new algorithms or modifying existing algorithms in computer vision for robustness, efficiency, and accuracy will be another critical part of this phase.

3. Evaluation: to measure the ability of technology to enhance mobility in both indoor and outdoor mobility courses with parameters such as the percentage of preferred walking speed, obstacle avoidance, hesitation, and self-reported mobility confidence.

It is hypothesized in this project that:

- Given the appropriate augmentations, AR can improve mobility in PLV.

- AR technology can improve users' experience in day-to-day activities related to mobility in PLV.

- The users' age, gender, spatial ability, and familiarity with technology affect mobility performance when the AR environment is used. From this perspective, AR technology targeting specific user groups may be more effective. 


\section{Progress so far}

In my first year of Ph.D., I have conducted a systematic literature review and a formative study. The review highlighted the need for a better understanding of PLV's requirements and formed the basis of some prototypes used in the following formative study.

\subsection{Formative study}

The purpose of this formative study is three-fold: to understand the nature of mobility problems experienced by PLV, to understand how and what they would like to have as vision enhancements, and to understand what they can or cannot see on HoloLens. This study consists of two parts: focus group study and follow-up one-to-one interviews.

\subsubsection{Focus Group Study}

To fully appreciate the unique challenges PLV face regarding orientation and mobility, we conducted four focus group discussions, each lasting about an hour. In total, 20 participants with varying visual conditions discussed challenging aspects of mobility in their day-to-day activities. Qualitative analysis of this focus group data revealed the impact of low vision on their mental and physical wellbeing, difficulties (e.g., walking into walls, not picking up steps or surface-level changes) and obstacles (e.g., bollards, overhanging branches, amongst others) they face in their environment, and what they think can potentially help (e.g., help with locating doors, coloring obstacles and drawing lines over glass doors to improve visibility).

\subsubsection{Face-to-face Interviews}

For the next stage, I developed seven prototypes of visual augmentations based on the literature and problems described in focus groups. Two prototypes use colors to convey distance by drawing colored meshes or by painting the environment with solid colors. Three prototypes overlay the edge images, high contrast images, and video feed of the live scene. Prototype with video feed allows users to zoom in using a tap gesture or verbal commands. One prototype recognizes objects in the scene using the YoloV3 algorithm [7] running on a remote laptop and provides verbal feedback in addition to drawing bounding boxes around them. Two prototypes draw lines on the walls to make them prominent or on the floor for users to follow to reach a predefined destination.
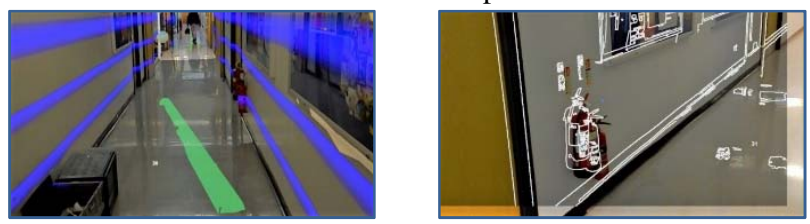

Figure 1: Example prototypes (left: lines on floors and walls, right: white edge overlays)

The prototype applications are shown to PLV in interviews, each lasting about an hour. During the interviews, participants put on HoloLens to observe the environment and walk around in a room and a long corridor and provided their feedback and opinions. The purpose of these interviews is to understand how different augmentations are perceived by PLV and to introduce the possibility of an AR environment to PLV so that they can make suggestions based on their mobility experiences. This is currently an ongoing study.

So far, the majority of participants find AR augmentations useful and suggested potential improvements (e.g. to augment only the immediate environment to avoid information overload, the need for faster processing to cope with dynamic objects). It was also learned that a participant with severe central vision loss due to end-stage retinitis pigmentosa could not see the augmentations.

\section{Conclusion}

While there are limitations with both current hardware and prototypes, the formative study shows promising signs that AR can enhance PLV's vision. As one participant commented: "[Seeing] the pictures, seeing your face, identifying it [things she could see without HoloLens], that was, to me, priceless!".

Currently, the prototypes have limitations such as low frame rates, delays in updating the virtual objects in dynamic environments, slow and lacking details from image filtering methods. Upon completion of formative study, the next steps in this project are to identify novel and potentially useful ways to use visual augmentations for PLV; to explore how to implement efficient interactions with virtual objects to customize and optimize them for different visual conditions and different environments; to explore different image style transformations to transform scenes to maximize visibility for PLV. Future generations of AR glasses could solve current hardware limitations.

I hope this research project can contribute to the AR research area by advancing the applicability of AR in a new domain as a mobility aid and uncovering new insights into the relationship between low vision conditions and AR environment. Most importantly, this can help unlock AR's potential as a life-changing technology for people with low vision.

\section{References}

[1] J Albouys-Perrois, J Laviole, C Briant, and A M Brock. 2018 Towards a multisensory augmented reality map for blind and low vision people: A participatory design approach. In Conference on Human Factors in Computing Systems - Proceedings.

[2] American Foundation for the Blind. Facts and Figures on Adults with Vision Loss. Retrieved from https://www.afb.org/researchand-initiatives/statistics/adults

[3] Alex D Hwang and Eli Peli. 2014. An Augmented-Reality Edge Enhancement Application for Google Glass. Optom. Vis. Sci. 91, 8 (2014), 1021-1030.

[4] Max Kinateder, Justin Gualtieri, Matt J. Dunn, Wojciech Jarosz, Xing-Dong Yang, and Emily A. Cooper. 2018. Using an Augmented Reality Device as a Distance-based Vision AidPromise and Limitations. Optom. Vis. Sci. 95,9 (2018), 727-737. M D Peláez-Coca, F Vargas-Martín, Sonia Mota, J Díaz, Eduardo Ros-Vidal, Maria Dolores Pelaez-Coca, Fernando VargasMartin, Sonia Mota, Javier Diaz, and Eduardo Ros-Vidal. 2009. A versatile optoelectronic aid for low vision patients. Ophthalmic Physiol. Opt. 29, 5 (2009), 565-572.

[6] Eli Peli, Gang Luo, Alex Bowers, and Noa Rensing. 2009. Development and Evaluation of Vision Multiplexing Devices for Vision Impairments. Int. J. Artif. Intell. Tools 18, 3 (2009), 365378.

[7] Joseph Redmon and Ali Farhadi. 2018. YOLOv3: An Incremental Improvement. arXiv (2018).

[8] Joram J. van Rheede, Iain R. Wilson, Rose I. Qian, Susan M. Downes, Christopher Kennard, and Stephen L. Hicks. 2015 Improving Mobility Performance in Low Vision With a DistanceBased Representation of the Visual Scene. Investig. Ophthalmol. Vis. Sci. 56, 8 (2015), 4802-4809.

[9] Sarit Szpiro, Yuhang Zhao, and Shiri Azenkot. 2016. Finding a store, searching for a product: a study of daily challenges of low vision people. UbiComp '16 (2016), 61-72. 